\title{
24. DISCUSSION FOLLOWING THE REPORTS \\ BY GREENBERG AND STECHER
}

(Wednesday, September 17, 1969)

\author{
Chairman: H. C. vAN DE HULST
}

Editor's remark: The material of this Discussion has been rearranged in five sections: 1. Grain Orientation; 2. The Diffuse Galactic Light; 3 . The Extinction Curve in the Far UV; 4. Formation and Destruction of Grains; 5. The OH Molecule. Except for rearranging, very few changes have been made.

\section{Grain Orientation}

Verschuur: Dr. Greenberg, what happened to the argument that alignment of grains required fields of the order of $10 \mu \mathrm{G}$ ?

Greenberg: During the past several years an enormous amount of new information has become available on the polarizing properties of elongated particles, and that, combined with my recent calculations on the particle orientation mechanism based on Jones and Spitzer's work (1967) gave a value of $8 \mu \mathrm{G}$ without stretching any one of the parameters. (Jones, R. V. and Spitzer, Jr. L.: 1967, Astrophys. J. 147, 943.)

Verschuur: Would you have to stretch the parameters to go down to $2 \mu \mathrm{G}$ ?

Greenberg: Yes, if I still demand the maximum ratio of polarization to extinction. By reducing $n_{\mathrm{H}}$ from $10 \mathrm{~cm}^{-3}$ to, say, $2 \mathrm{~cm}^{-3}$ the required field is down by a factor of 2. If I further allow the ratio of polarization to extinction to be 0.025 rather than 0.06 , then a magnetic field of $2 \mu \mathrm{G}$ would be sufficient. I would like to add that, as stressed earlier by Woltjer (p. 195), the optical polarization gives essentially the squared field. Perhaps variations in the direction of the magnetic field reduce the linear average, which you see in the Zeeman and in the Faraday measurements, while a sufficiently large squared average remains to produce the optical polarization. Also, a small contribution by super-paramagnetism could decrease the field requirement further.

Kurt: The grain charge depends on the nature of the dust particles (metallic or dielectric) on the UV radiation and on the interstellar electron density. For the specific conditions in interstellar space we may have both positive or negative charges. What is your opinion, Dr. Greenberg?

Greenberg: I think that the dust is mainly negatively charged. If there is an electric or (because of rotation) a magnetic dipole, there are other forces, but I do not expect that they are large enough to affect the orientation.

Van de Hulst: The charge is also important in connection with the drag between gas and dust.

Dolginov: If the grains are charged, but the center of charge distribution is not the 
center of gravity because the grain has a complex shape, then an electric dipole results and electrical forces occur when the grain moves with respect to the interstellar magnetic field. If the dust and the magnetic field move with respect to each other with differential velocities of at least $1 \mathrm{~km} \mathrm{sec}^{-1}$, the electrical forces are large enough to destroy the orientation of the particles. There is also another possibility to orient the grains: place the dust particles in a gas flow; if the relative flow velocity is about $10 \mathrm{~km} \mathrm{sec}^{-1}$, then one can calculate (Dolginov, 1968) that within $10^{3} \mathrm{yr}$ the grains are oriented perpendicular to the flow velocity. It takes about $10^{6} \mathrm{yr}$ for the grains to attain the flow velocity, after which the orientation will be random. This process of grain orientation (originally proposed by Gold) will work in the solar wind and in stellar winds. A somewhat similar, but quantitatively (usually) somewhat less important process is that of particles placed in an anisotropic radiation field. [Dolginov, A. Z.: 1968, Dokl. Akad. Nauk SSSR 179, 1070 (translation: 1968, Soviet Phys. Dokl. 13, 281).]

Greenberg: The grains are slowed down in about a third of a parsec for a gas of any density. They do not maintain a sufficient relative velocity unless there is a driving mechanism. Recently Purcell (1969) has looked into the polarization produced by grains driven through a gas by anisotropic stellar radiation. The result was not enough to account for the polarization. One should combine all proposed mechanisms and see whether they combine to give the observed amount of polarization relative to extinction, or whether they detract from each other. I think that the magnetic field by itself is sufficient. [Purcell, E. M.: 1969, Physica 41, 100 (Proceedings International Conference on Laboratory Astrophysics, ed. by J. Rosenberg, Lunteren, 1968).]

\section{The Diffuse Galactic Light}

Rozhkovskii: At the Astrophysical Institute of the Kazakian Academy of Sciences, we are making photometric studies of the diffuse galactic radiation. The methods of our observations and other details of the investigation are described elsewhere (Rozhkovskii 1969a, b). My remarks concern only the albedo $\gamma$ of the interstellar grains. Our observations show $0.3<\gamma<0.6$, with a probable error of 0.3 accounting for uncertainties in the observational data. However, even with so large an uncertainty it seems that the optical properties of grains are sufficiently different from those of perfect dielectric particles. Our result differs significantly from that of Witt (1968), who found that $\gamma$ must be extremely close to 1 (about 0.98 ).

The discrepancy between the two results has a simple explanation. For the interpretation of his observation, Witt used Henyey and Greenstein's (1941) theoretical treatment. Their formulae are only approximate. I found, however, a rigorous analytic solution of the equation of transfer in Eddington's approximation in the special case when $\gamma=1$ and the scattering is isotropic. It appears that the predicted intensity is higher in our solution than in that of Henyey and Greenstein. Therefore Witt found too high an albedo for the grains compared to what actually is required. (Rozhkovskii, D. A.: 1969a, Vest. Akad. Nauk Kazakhskoi SSR 9, Rozhkovskii, D. A.: 1969 b, 
Vest. Akad. Nauk Kazakhskoi SSR 7, 63; Witt, A. N.: 1968, Astrophys. J. 152, 59; Henyey, L. G. and Greenstein, J. L.: 1941, Astrophys. J. 93, 70.)

Van de Hulst: You have been speaking of the differences between two computed curves. In Leiden de Jong and I made a similar study by numerical means rather than by analytic solution of the equation of transfer (van de Hulst and de Jong, 1969) and we disagree in the same sense as you do with Witt's conclusion regarding $\gamma$.

Let me mention that at the same observatory Glozhkov and others have done much work on reflection nebulae (e.g., Glozhkov, 1968) but nobody has been asked to talk about those here. [van de Hulst, H. C. and de Jong, T.: 1969, Physica 41, 151 (Proceedings International Conference on Laboratory Astrophysics, ed. by J. Rosenberg, Lunteren, 1968); Glozhkov, Yu. I.: 1968, Trudy Astrofiz. Inst. Akad. Nauk Kazakhskoi SSR 11, 57.]

\section{Extinction Curve in the Far UV}

Kurt: I should like to have a discussion on Stecher's newest measurements of the extinction curve in the UV.

Stecher: The curve you mentioned has been published recently (Stecher, 1969). Let me summarize this paper. With an Aerobee rocket I obtained spectra between $1150 \AA$ and $4000 \AA$ of $\zeta$ Persei and $\varepsilon$ Persei. Figure 1 presents the difference in magnitude ( $\zeta$ Persei $-\varepsilon$ Persei), for each $2 \AA$ interval. The differences have been multiplied by a

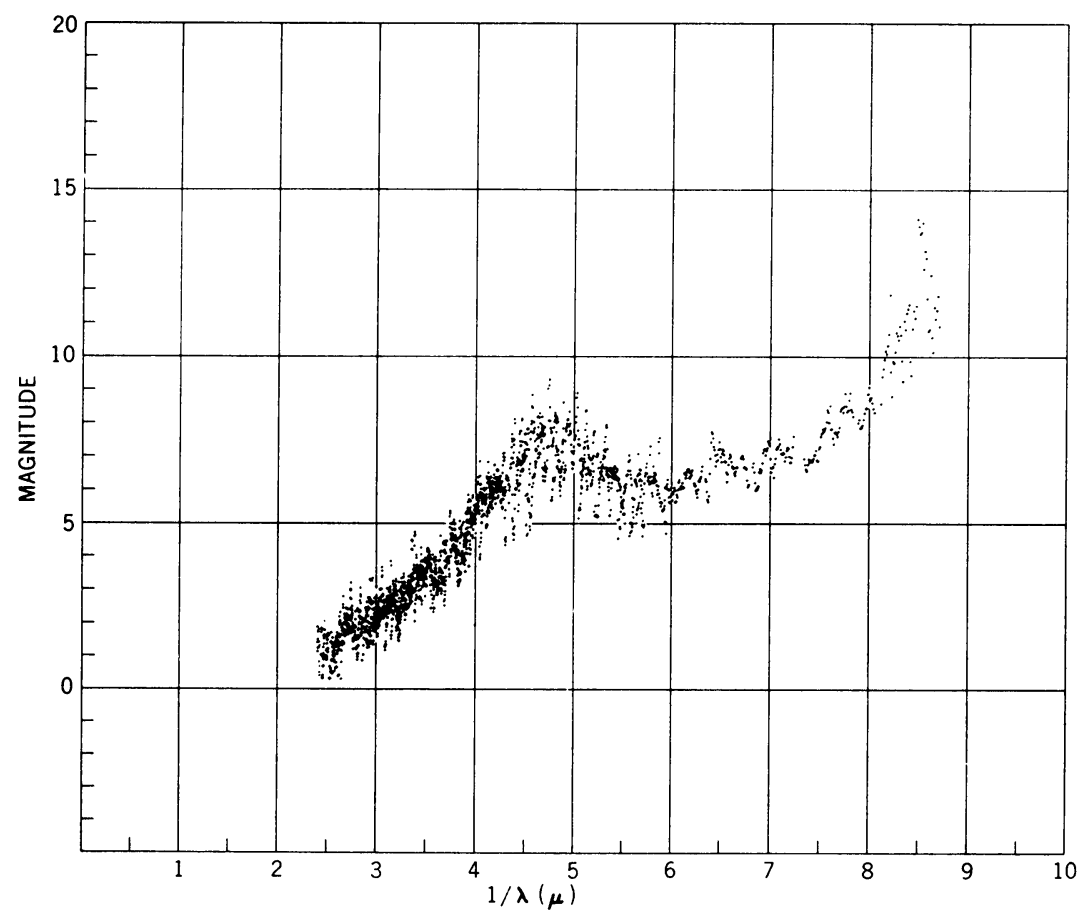

Fig. 1. (See the remark by Stecher.) Interstellar extinction in magnitudes as a function of inverse wavelength determined from $\zeta$ and $\varepsilon$ Persei. The curve is normalized to $B-V=1 \mathrm{mag}$ and $V=0$. 
factor of 3.57 to normalize the observations to $B-V=1$. The two stars are quite close in visual magnitude and therefore the curve goes through $V=0$ without any additional normalization. When a small correction for the geocorona Ly- $\alpha$ radiation is applied, the curve will show a very large extinction near $8.22 \mu^{-1}$ due to the interstellar $L y-\alpha$ absorption line. Only the region within $100 \AA$ of $L y-\alpha$ is affected by this. The difference in spectral type is of minor importance ( $\zeta$ Persei is BlIb and $\varepsilon$ Persei is B0.5V).

The present extinction curve may be considered a confirmation of earlier work (Stecher, 1965). Its main characteristic is the hump at about $4.7 \mu^{-1}$, which is quite clearly present. Stecher and Donn (1965) and Wickramasinghe and Guillaume (1965) have pointed out that a transition in graphite would give rise to a similar feature. At the shorter wavelength another material is necessary if graphite is assumed. Atomic and molecular gases (Stecher and Williams, 1969) as well as coated particles could serve as such. Some minor features are atomic and are probably circumstellar. (Stecher, T. P.: 1965, Astrophys. J. 142, 1683; Stecher, T. P.: 1969, Astrophys. J. Lett. 157, L125; Stecher, T. P. and Donn, B.: 1965, Astrophys. J. 142, 1681 ; Stecher, T. P. and Williams, D. A.: 1969, in preparation; Wickramasinghe, N. C. and Guillaume, C.: 1965, Nature 207, 366.)

Kurt: Dr. Stecher, in the slide [Figure 1. (Ed.)] we can see an absorption line at about $1538 \AA, 1408 \AA$ and $1178 \AA$. Is this real or is it an instrumental effect?

Stecher: There are several lines, but I believe them to be circumstellar. For instance Civ at $1549 \AA$ is present.

Greenberg: Figure 2 shows some model calculations I made. The dash-dot curve is pure $\mathrm{H}_{2} \mathrm{O}$-ice. Characteristically all dielectric-grain models show some absorption

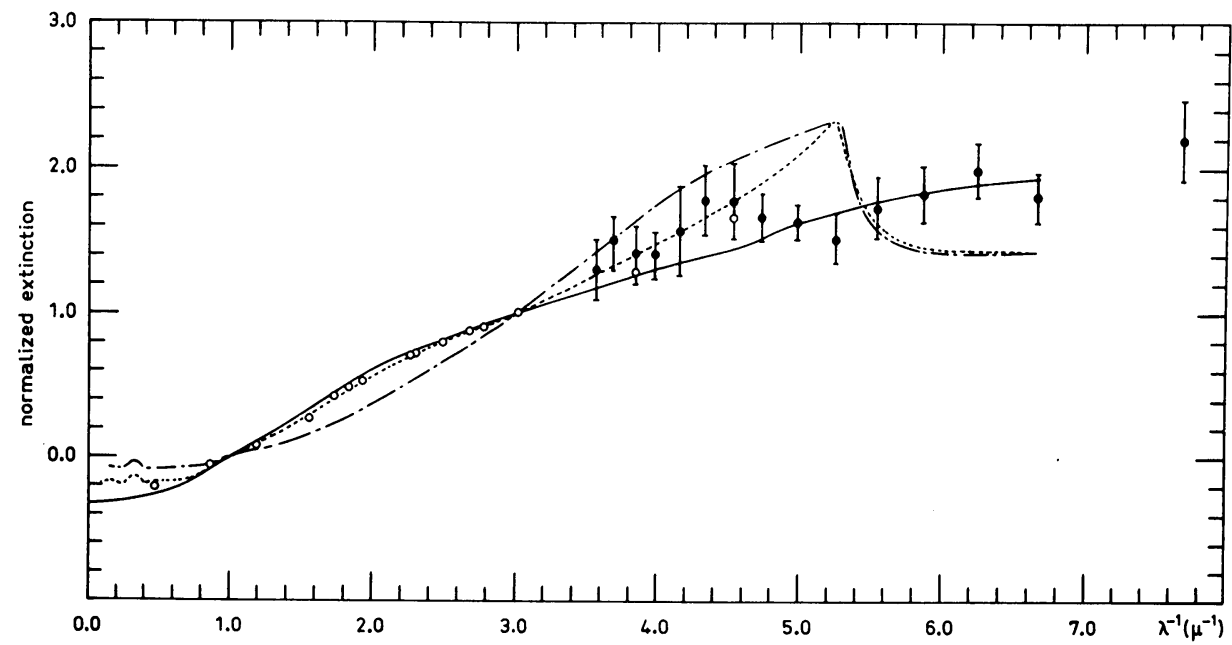

Fig. 2. (See the remark by Greenberg.) The solid curve shows the extinction by a graphite core (radius $0.054 \mu$ ) with a dielectric $(m=1.33-0.05 i)$ mantle (radius $0.16 \mu)$; the dashed curve is for a 'dirty-ice' mantle, whose index of refraction varies according to the best available measurements; dash-dot curve is for extinction by a solid 'dirty-ice' grain (no graphite core) with a radius of $0.16 \mu$.

The rise and drop in the latter two curves is caused by an absorption edge for the ice. 
at about $5 \mu^{-1}$, so that the drop in the curve is easy to make with dielectric particles. The drop does not reproduce the absorption band exactly, but one could use other molecules to smooth the effect a little. Regardless of whether we have the graphite core or some dielectric core (like silicates) it is always a problem to find a material which causes a continuous rise in extinction after the drop. This situation has to do with the fact that if an absorbing particle has the size of the wavelength, its extinction continues to drop. But what happens when the surfaces of particles are not smooth? I have shown that irregular particles will smooth out the kinds of details shown in Figure 2 because they would give rise to an effective absorption in the wavelength region before the onset of real absorption. The result is that real absorptivity would not show up strongly. If the particles are rough, then all spectral characteristics would have to be based on more elaborate discussions of the scattering and absorbing properties of particles of that nature. Stecher pointed out that graphite particles could give the observed hump, but the graphite model alone does not produce the continued rise in the extinction observed beyond $5 \mu^{-1}$, and Stecher needs to put in another material as well. Further any model with enough graphite to produce the hump does not satisfy the observed wavelength dependence of polarization. We also note that graphite particles cannot be oriented by the magnetic field; therefore the polarization has to be produced by that extra ingredient alone. If you add enough of that material (but not enough to obscure the hump), then you cannot get the observed ratio of polarization to extinction, regardless of what the mechanism of orientation is.

Shulman: Can the rise for $\lambda^{-1}>5 \mu^{-1}$ be explained by the presence of large organic molecules?

Stecher: A large molecule with benzene rings in it would probably have the sort of extinction required. The amount of extinction yields at once the amount of $\mathrm{H}_{2}^{+}$in the Galaxy; one obtains $n\left(\mathrm{H}_{2}^{+}\right)=2 \times 10^{-4} \mathrm{~cm}^{-3}$.

Shulman: I should like to suggest that large molecules can exist in grains. Let us consider a grain covered by the simplest radicals mentioned here $(\mathrm{CH}, \mathrm{OH}$, etc.). Suppose that the grain is exposed to cosmic rays, then it is easy to show that the energy input is of the order of $10^{-14} \mathrm{eV} \mathrm{sec}-1$ per radical. It is a well-known chemical fact that synthesis of heavy molecules can take place in solids induced by radiation. The rate of radiative synthesis depends on many conditions such as temperature, structure, chemical composition and others. Gains are found ranging from $10^{-2}$ to 10 reactions per eV. The exact value in the case considered here is unknown. If we estimate one reaction per $\mathrm{eV}$, almost any radical will take part in a chemical reaction in a time of the order of $10^{7} \mathrm{yr}$. Actually not only radiative synthesis occurs, but also radiative destruction of complex molecules. However, chemical experiments have shown that radiative synthesis is more probable. It is, for example, an interesting fact that solid methane $\left(\mathrm{CH}_{4}\right)$ at $77 \mathrm{~K}$ when exposed to gamma radiation, transforms into oil with a large molecule of the form $\mathrm{C}_{20} \mathrm{H}_{n}$ where $n$ may have various values. So we can expect more complex molecules than $\mathrm{CH}$ (and similar ones) on interstellar grains. Can anybody tell me whether it is possible to detect such molecules?

Van de Hulst: I might answer by saying no, because, first of all even if you had 
these molecules nicely chemically isolated in a gas, then you would have difficulty recognizing their spectra among those of similar molecules. But here, where they are imbedded in a particle, the absorption of the bulk material might show up as a reduction (thereby an apparent emission) in the star spectrum. I would think that a chance of a positive identification for such molecules is very small.

Field: I disagree with van de Hulst on the question of whether or not one might identify organic compounds. In particular, several people who discussed the relationship between the unidentified lines and the grains suggested that the width of the lines can be interpreted as due to molecules imbedded in the grains and interacting strongly with the grain matrix. F. M. Johnson, in the U.S.A., investigated possible identification of these lines with heavy organic molecules (Johnson, 1967). Also in response to Shulman's remark, we should consider the effects of X-ray emission by discrete sources, such as Sco XR-1. If Shulman is correct, one might expect differences in the grain properties near X-ray sources, because the X-ray quanta have similar effects to those of cosmic rays in changing the chemical compounds in the grain. One might, for example suggest observing the unidentified feature at $\lambda 4430$, in the neighborhood of such X-ray sources. I have been considering a model for explaining the intense interstellar calcium lines in the spectrum of Sco XR-1 by the removal of material from grains by X-ray interaction. I can explain the calcium in the gas phase as due to ejection from the grain by $1-\mathrm{keV}$ photons which generate very energetic electrons within the grains. It is interesting that in Scorpius the calcium in the gas phase is generally 1000 times less than would have been predicted from solar abundances. We therefore need to remove only about 1 per cent of the calcium locked up in the grains (if that is, in fact, where the missing calcium is), in order to explain the observations in the Scorpius region. [Johnson, F. M.: 1967, in Interstellar Grains (ed. by J. M. Greenberg and T. P. Roark), Office of Technology Utilization, National Aeronautics and Space Administration, Washington, D. C.]

Shulman: I agree with Field, because only the total energy input has significance in such a process, but not the nature of the radiation. X-radiation and cosmic protons give the same effect.

Greenberg: I agree that the unidentified lines may be one of the keys to unlocking at least one of the problems of the interstellar grains. I have done a calculation on the polarization of an absorption band in the grain material. Historically the consideration of producing diffuse lines by atoms or molecules in grains goes back to our Chairman (H. C. van de Hulst). Many years ago I myself tried calcium as a source of $\lambda 4430$. The problem is, however, that any kind of absorbing material whose general optical characteristics give rise to the observed extinction and polarization, does not give an absorption line at $4430 \AA$ but rather a dispersion curve (i.e., a profile with an emission wing at shorter wavelengths and absorption at longer wavelengths). This is a general and unavoidable result. If you put the material in a metal grain which is also already absorbing, the absorption line is relatively reduced or you do not find the line at all. From an observational point of view it is very difficult to measure the exact shape of the $\lambda 4430$ line because of all the background hash. However, several people have 
recently claimed that the $\lambda 4430$ line is indeed an asymmetric feature with a strong emission wing at the shorter wavelength. I refer specifically to Seddon at Edinburgh, but Walker at Victoria also proposed a rather significant emission wing at the shorter wavelength. Some time ago I made a calculation in which I tried to eliminate the emission wing by varying particle size distribution, since the observations of that time said that $\lambda 4430$ was symmetric. But, whatever I tried, the absorption band always turned out to be of the dispersion type. In my most recent calculations for cylinders, I make a prediction of the polarization of $\lambda 4430$. From an observational point of view the polarization is independent of the star and is therefore a more suitable source of unambiguous information than the shape of the absorption band. The key to unlock the puzzle of the grains may well be to look at polarization in the diffuse lines. The shape of the polarization can distinguish whether the particles are dielectric or metallic.

Field: I want to emphasize that $\mathrm{NH}_{3}$ has a photodissociation edge at exactly $4.7 \mu^{-1}$. Is it possible to explain the bump as a rise with wavelength in the extinction coefficient at the long wavelength side of the bump, followed by a drop when one goes over the absorption edge of $\mathrm{NH}_{3}$ ?

Greenberg: On the curve that I presented (Figure 2) I included only ice. Including other molecules would shift the hump. I discovered, however, that I still had difficulty getting the extinction to rise in the far UV beyond the drop. Possibly surface roughness will modify the sizes of the particles somewhat and still maintain a continued rise in the extinction curve. A purely theoretical but not very quantitative calculation indicates the possibility of such a rise by using dielectric material.

I should also point out that, thus far, this bump has only been confirmed in rather nearby, very hot stars. I would like to see it confirmed more generally. Is it possible that this hump is a selection effect? After all, these are rather small extinctions, therefore relatively local. I have still another point. There is an old anomaly in the near UV extinction of $\theta^{\prime}$ Orionis. The observations in the far UV by Carruthers indicate that the extinction curve levels off instead of continuing to rise, a behavior which agrees with the classical result. I would like to see the shape of the $4.7 \mu^{-1}$ hump for this star. It would help us to find out what characteristics, such as grain size, can do to absorption features.

Lynds: Your criticism of the UV portion of the extinction curve holds for the entire extinction curve, which might well represent only the nearby region of the Galaxy.

Field: I did some calculations in 1963 that showed that in the presence of $\mathrm{NH}_{3}$, the curve will rise smoothly, then drop suddenly at $4.7 \mu^{-1}$. After this it either rises or stays constant, I do not remember which. I therefore wonder whether Dr. Greenberg does or does not assign a specific meaning to the $4.7 \mu^{-1}$ ?

Greenberg: No, I don't.

Field: I do not understand that reply because the dissociation potentials of the molecules are determined very well in the laboratory. The limit for $\mathrm{H}_{2} \mathrm{O}$ is $1500 \AA$; for $\mathrm{CH}_{4} 1350 \AA$; and $\mathrm{NH}_{3}$ gives the only potential that fits. On the other hand, we hear from Stecher that the hump is caused by graphite. Therefore, I am lost. 
Van de Hulst: In order to identify the unknown constituent the theorists should settle first the question of whether to look at the frequency of $4.7 \mu^{-1}$, or whether to look at the combined feature of hump and valley, saying that it all belongs to the same resonance. I have always had the feeling that once you have the right frequency then a second uncertainty may show up because many chemicals will fit into a particular feature. Does Dr. Greenberg think that is true?

Greenberg: Yes. To begin with we must study the effects of spectroscopic candidates as they show up in small, solid particles. First, the observed feature is very broad. Second, if it is due to something in the interior composition of the grain, the shape of the feature would be size-dependent. Therefore, to attribute a specific feature to something at a precise frequency is a bit premature. There are many dielectric materials which have absorption edges around this frequency. For this reason, I prefer to make a model that fits the whole curve, including the right shape of the hump. Experimental studies of absorptivities of various mixtures of the simple $\left(\mathrm{H}_{2} \mathrm{O}, \mathrm{NH}_{3}, \mathrm{CH}_{4}\right)$ molecules should be made.

Field: I suggest that the original van de Hulst model, which has a well-defined composition, including some $\mathrm{NH}_{3}$, should be tested by calculations.

Van de Hulst: The model was only a guess.

Stecher: I think that with a reasonable size distribution, a graphite particle will fit the data, out to $\lambda^{-1} \approx 6 \mu^{-1}$; beyond that, graphite does not provide enough extinction.

It is my impression that the uniformity of the extinction curve in different directions is quite remarkable. This may be partly because of observational selection of close-by clouds; but on the other hand we have variations in gas density of a factor of 10 to 100 . The accretion should be proportional to the square of the density and therefore, the grains should grow quite rapidly to large sizes and give only geometric extinction. But we still observe the $1 / \lambda$ variation in every direction.

Van de Hulst: Like Stecher, I was always impressed by the rather amazing uniformity which still holds in spite of the differences in the extinction curve you see from place to place. I am somewhat relieved by the new UV observations in the following respect. In the old observations of the extinction curve, where $\lambda^{-1}<3 \mu^{-1}$, the most obvious way to extrapolate was to have a maximum near $\lambda^{-1}=4 \mu^{-1}$, which puts the main importance in fitting not on the material of the grains but on their sizes. Now we have new observations which go to $\lambda^{-1}=9 \mu^{-1}$ and the gross features of the extinction curve should correspond more to a smooth curve with a maximum near $\lambda^{-1}=8$ or $10 \mu^{-1}$, leveling off beyond that. This automatically means smaller grains, relatively more absorption in the visual range, and a less sensitive dependence on the assumed size. So this relieves the difficulty, but of course it does not solve the complete problem before you have solved all details of the wiggles, which are definitely dependent on composition.

Shklovskii: Many times in the past I have drawn attention to one remarkable fact. We know that the density of interstellar grains is about one per cent of the density of interstellar gas. On the other hand, the density of heavy elements in interstellar gas relative to hydrogen is also close to one per cent. Since the grain particles consist of 
heavy elements I have the impression that perhaps 50 per cent of the heavy atoms in the interstellar medium is condensed in dust and 50 per cent is in the gas. It is a very unexpected situation and may not be explainable by chance. I will not attempt to give an answer; but perhaps here is an argument in favor of the origin of grain particles in the atmospheres of red giants; maybe so, maybe not.

\section{Formation and Destruction of Grains}

Van de Hulst: I had one further question on the dust from stars. Like Greenberg I find it difficult to believe that most of the dust comes from stars. Linking up with the data on the mass loss of stars (p. 273 and p. 289) I made this calculation.

$$
\begin{aligned}
& \text { Dust present in the Galaxy } 10^{11} M_{\odot} \times 10^{-4}=10^{7} M_{\odot} \\
& \text { Rate of supply from stars } 1\left(M_{\odot} \mathrm{yr}^{-1}\right) \times 10^{-1} \times 10^{-2}=10^{-3} M_{\odot} \mathrm{yr}^{-1} \\
& \text { Supply time } \quad=10^{10} \mathrm{yr} \text {. }
\end{aligned}
$$

The factor in the first line is the estimate that about $10^{-2}$ of the total mass of the Galaxy is gas, about $10^{-2}$ of the gas is condensed in dust. The factors in the second line signify that perhaps one kind of star out of ten supplies dust and that by the abundances only $10^{-2}$ get into the grains. The $10^{10} \mathrm{yr}$ seems to me too long to pretend that all dust is supplied in this manner. I know other people have made such calculations and have reached a different conclusion, but $\mathrm{I}$ do not know if anybody here wants to defend that position.

Greenberg: I agree with such a calculation. However, ejection from stars may be one of the mechanisms for creating growth nuclei (grain seeds) in interstellar space. There is one other mechanism for dust production that I should mention, although I believe it is less effective by orders of magnitude. In this process, proposed by Dorscher (1967), particles are ground in a planetary system around a star and are ultimately ejected, as happens to the particles in the solar system.

In general, I think that grain seeds do not form directly in interstellar space, but that little particles are injected into space onto which molecules or gas atoms can come and stick. (Dorscher, J.: 1967, Astron. Nachr. 290, 171.)

Stecher: I am an author of a paper which purports to get enough grains from stars. We used Schwarzchild's value of $10^{9} \mathrm{yr}$ for turnover time in the interstellar material and considered the grains to be refractory. With the new sputtering rate the grains are not destroyed until they actually go into the stars. Under these conditions $10^{9} \mathrm{yr}$ seems to be long enough.

Weymann: Dr. Greenberg, there are many places now where there is a suspicion of coexistence of dust and $\mathrm{H}$ II gas. Can you bring us up to date on what is known about destruction of dust by $\mathrm{O}$ stars? I would like to know particularly how the destruction rate depends on the energy of the photons. Is it just a matter of total energy density upon the surface, or do we need to know more details about the spectral distribution of the radiation? Also, can you give a simple criterion for deciding when the grains are more rapidly destroyed by hot gas than by the radiation field? 
Greenberg: I think it is well established that in HII regions there is dust; there is certainly reflected continuum radiation in the Orion Nebula (O'Dell et al., 1966). Atomic collisions at $10000 \mathrm{~K}$ are exceedingly inefficient in knocking anything off, for the threshold for sputtering from experimental results is, I believe, something like $10 \mathrm{eV}$. Energies of a few $\mathrm{keV}$ are required for an efficient sputtering process. As far as the UV radiation is concerned, it may raise the temperature of the grains. You need grain temperatures of $90 \mathrm{~K}$ or so in normal stellar surroundings to evaporate ice off the grain. If the material is somewhat more refractory than ice, you would not evaporate it at all. But to get $90 \mathrm{~K}$ temperatures you have to be very close to the star; otherwise T is considerably lower. (O'Dell, C. R., Hubbard, W. B., and Peimbert, M.: 1966, Astrophys. J. 143, 743.)

Ozernoi: Are there any differences between the properties of interstellar grains, in particular with respect to their chemical composition, in the direction of the galactic center and in the opposite direction? And, similarly, what are the properties of the dust grains in radio galaxies?

Van de Hulst: When you investigate clouds in the direction of the galactic center, you still talk about clouds which are very nearby, generally, and not at the galactic center.

\section{The Gas/Dust Ratio}

Habing: There have been several investigations in which one compares dust clouds with $21-\mathrm{cm}$ observations. Implicit in these discussions has been the assumption that everywhere the ratio of the number of grains to the number of hydrogen atoms in the line-of-sight is constant. To me, such an assumption seems questionable. Could Dr. Greenberg comment?

Greenberg: I think that there is evidence for an average or general correlation of hydrogen and dust but I am not aware that the local correlation needs to follow. If the dust grows out of the gas and there are no effective mechanisms to bring about a subsequent separation, then we would find an excellent correlation between hydrogen and dust. Now we ask: what happens in very dense clouds where there is apparently an anticorrelation? Does it occur because of a separation of gas and dust after dust formation or perhaps because the gas is in an at present unobservable form - say molecular hydrogen? But if the dust does not exclusively grow from the interstellar gas we must ask instead: Why should there be a correlation? As a matter of fact, if Herbig's ideas have some significance, it is possible that one could have molecules and dust grains thrown into clouds from a star formation process without the existence of a great amount of hydrogen. We really must therefore find out what is in those dust clouds. There may be a correlation of dust and gas even if there is no correlation of dust and hydrogen.*

Habing: So the conclusion that if one does not observe any excess hydrogen at the

* (Note added in proof.) There are now several dust clouds known that show up in the $21 \mathrm{~cm}$ line profiles. The interpretation is uncertain - either the atomic hydrogen is deficient or it is cool. (Heiles, C. E.: 1969, Astrophys. J. 156, 493; Sancisi, R. and Wesselius, P. R.: 1970, Astron. Astrophys. 7, 341.) 
position of a dust cloud, therefore all the extra hydrogen is molecular is not necessarily true?

Greenberg: There is no firm theoretical basis for this assumption but it is a possibility.

Van de Hulst: From direct photographs the dust clouds occasionally have very sharp edges. I have never heard of a mechanism working on the dust that can create such sharp edges. Such mechanisms, however, do exist for the gas, and this has always been a convincing argument for me, that dust and gas go together.

Zimmermann: I have calculated dust motions during a cloud collision (Zimmermann, 1968). During the collision the heavy particles move into the inner parts of the newbuilt cloud and take the form of a dense sheet. They remain there during the expansion period. So at the end, when pressure equilibrium with the intercloud medium is reached, we have the heavy dust particles in the middle of the configuration while the light dust particles are distributed more or less uniformly among the gas of the cloud. Therefore we have a dynamical separation process of particles and dust. (Zimmermann, H.: 1968, Astron. Nachr. 290, 193.)

Field: If a separation occurs between gas and dust then clouds exist without the cosmical abundance, i.e., with large amounts of heavy elements and no hydrogen. How does one then explain that there exist no stars with such a composition?

Greenberg: Clouds without hydrogen would have smaller masses than are obtained by assuming a standard hydrogen-to-dust ratio. Maybe the conditions for star formation are then not favorable.

Field: I should have thought that clouds of dust would collapse very easily under their own self-gravitation and external radiation pressure, as the internal pressure is negligible.

Van Woerden: I have a small comment about the gas/dust ratio. In considering the lack of correlation between gas and dust, one should not only take into account the possibilities for molecular hydrogen or even for abnormal abundances, but one should also consider the effect of possible variations of the gas temperature. In the milder cases of non-correlation, such as that in the Taurus region discussed by Garzoli and Varsavsky (1966), assumption of a gas temperature below $125 \mathrm{~K}$ restores the normal gas-to-dust ratio, as has been shown by Sancisi and Wesselius (op. cit.). (Garzoli, S. L. and Varsavsky, C. M.: 1966, Astrophys. J. 145, 79.)

Field: I have a question for Dr. Lynds. What is the evidence about the existence of dust in regions where the gas density is very low, for example what do we know about extinction in stars, say 100 pc away, and with very weak interstellar lines?

Lynds: All I know is the old work on the general correlation of the strength of the calcium lines with extinction. There is a much wider scatter in this relation than can be interpreted as errors in observations. I do not know what the upper limit you refer to would be.

\section{The $\mathrm{OH}$ Molecule}

Van de Hulst: Dr. Weaver has been challenged to give his view on the future of all the $\mathrm{OH}$ observations. 
Weaver: I certainly do not know what the future of all the $\mathrm{OH}$ observations will be, but there are some aspects of the $\mathrm{OH}$ problem that perhaps should be discussed here. One thing that has impressed me very much in looking at the $\mathrm{OH}$ absorption observations in conjunction with neutral $\mathrm{H}$ absorption observations is that if you observe an $\mathrm{OH}$ absorption line and a neutral $\mathrm{H}$ absorption line they have the same velocity, within observational error. I would suggest that this evidence indicates that there must be a very close association of the $\mathrm{OH}$ molecule formation mechanism and the concentration of the neutral $\mathrm{H}$ seen as a 'cloud' in a particular direction. The aspect of the $\mathrm{OH}$ that is rather less well-correlated with the $\mathrm{H}$ is the line width. One would predict on any reasonable basis that the $\mathrm{OH}$ line is much narrower than the $\mathrm{H}$ line. In fact, in many instances the half-width of the $\mathrm{OH}$ line is very much greater than the half-width of the $\mathrm{H}$ line. There is a very peculiar situation in regard to the velocity distribution of the $\mathrm{OH}$ molecule in the cloud seen in absorption. These properties, it seems to me, are directly related to some of the aspects of the Symposium topics.

Greenberg: If, in absorption, the $\mathrm{OH}$ line is wider than the $\mathrm{HI}$, does this not indicate that there is no thermal equilibrium?

Shklovskii: The velocities are not thermal, but result from macroscopic motion.

Menon: The most conspicuous case is the absorption toward the galactic center. The $\mathrm{OH}$ absorption is the strongest in those features which are the widest in $21 \mathrm{~cm}$. I do not know how that observation fits with your anti-correlation.

Weaver: These very broad features Menon mentions are examples of another special class of $\mathrm{OH}$ sources. $\mathrm{OH}$ is a molecule that seems to disobey all standard rules and to appear spectroscopically in almost any form that one can imagine. I think the $\mathrm{OH}$ features in the direction of the galactic center occur in very special structures in the interstellar medium in which molecules are very numerous. In these regions towards the galactic center one finds $\mathrm{NH}_{3}, \mathrm{H}_{2} \mathrm{CO}$, and so on. The areas are small, diameters less than about $10^{\prime}$. They must represent regions where molecule formation goes on very strongly. They are the best place in the sky to look for new molecules. But I really did not have these strange spots in mind when I spoke about the relationship of linewidth between $\mathrm{OH}$ and $\mathrm{H}$. The abnormality in $\mathrm{OH}$ widths occurs in the more common sources seen in various positions in the galactic plane. I was trying to confine my remarks to the general distribution of $\mathrm{OH}$ rather than to specialized regions.

Shklovskii: What is the situation now for the relative intensity of the different absorption components of the $\mathrm{OH}$ line? Do they satisfy the Boltzmann ratios?

Weaver: They tend to show non-LTE ratios. In some instances they may show almost-LTE ratios; whether this appearance is real or accidental, I do not know.

Varshalovich: Usually the description of the state of atoms and molecules in space takes into account particle density distribution, velocity distribution, and the degree of ionization and excitation. But spin states of particles have never been considered. Apparently spins were assumed to be oriented at random and to play a negligible role in the problems considered. This assumption is correct for the conditions here on Earth. But it does not necessarily hold in the interplanetary and in the interstellar medium, where there is no thermodynamic equilibrium. In an analysis of physical 
conditions in various astrophysical objects I have shown (Varshalovich, 1965) that the alignment of spins should be a rather common phenomenon in the Universe. I considered two possible mechanisms of orientation of spins: (1) equilibrium orientation by a magnetic field; and (2) non-equilibrium dynamical orientation by directed fluxes of photons or particles. Magnetic orientation is not important in outer space, because $\mu B \ll k T$. But the second mechanism is important. Resonance scattering of directed fluxes of nonpolarized radiation can be an effective mechanism when a magnetic field also is present. Necessary for the process to operate are the following conditions: (a) the interaction of particles with radiation is greater than the interaction with other particles; (b) the radiation is anisotropic. Spin alignment due to resonance scattering of unpolarized radiation has been observed in the laboratory by $\mathrm{A}$. Kästler and others. In the laboratory the system quickly relaxes to the isotropic equilibrium state. At the same time, there are vast regions in outer space where all the necessary conditions are fulfilled and spin alignment is maintained permanently by nature itself: nebulae in the interstellar medium, upper layers of stellar atmospheres, comets, and so forth. These objects have in common that the radiation fluxes are rather large and essentially anisotropic (because the optical thickness is small $\tau \lesssim 1$ ), and interparticle collision relaxation is negligible, because the density is small.

Optical properties (extinction and refraction) of the medium which contains spin aligned particles depend strongly on the direction of observation and on the polarization. Both decrease and increase of the optical thickness of resonance radiation are possible. If the ground state has a fine or superfine structure, spin alignment may give inverse populations of the upper state magnetic sublevels relative to the corresponding lower state ones and can lead to amplification of resonance radiation. Initially unpolarized radiation, while propagating through such a medium, acquires linear polarization. Spin alignment may substantially affect resonance radiation transfer. One should take it into account in plotting the curve of growth, by introducing the corresponding changes in the optical thickness. In some cases it may give an appreciable correction for the derived numbers of atoms and molecules; i.e., it may change the derived chemical composition, degree of ionization, and degree of excitation. Moreover, analysis in terms of spin alignment gives quite new information on the anisotropy of the prevalent physical conditions and, particularly, on the anisotropy of the radiation field. Finally, the fact that spin alignment produced by optical pumping depends on the direction of the field makes it possible to determine this direction in a given region of space. [Varshalovich, D. A.: 1965, Astron. Zh. 42, 557 (translation: Soviet Astron. 9, 442).]

Van de Hulst: It is obvious that if alignment could be observed, it would indeed give very good clues. Do you have a particular molecule in mind where it would be observable?

Varshalovich: As an example, I have considered in detail the spin alignment of $\mathrm{NaI}$ due to the pumping of solar radiation in the head of a comet.

Shklovskii: It is a long way from the physical considerations to astrophysical 
applications. The physical side is evident, but in astrophysical conditions the mean free path of the resonance quanta is short, and it is very difficult to obtain more or less strong anisotropies in the radiation field. Perhaps sometimes the situation is favorable for spin orientation, but in the general astronomical situation it is difficult to fulfill the necessary conditions.

Van de Hulst: At the end of the discussion I should like to add a few comments, because I often find that the history starts too late, and that earlier contributions are not mentioned at all. To my knowledge, the first discussion of growth of grains due to condensation by interstellar gas was by Lindblad in about 1935; the refinement which Oort and I tried to make in 1946 takes account of the chemical composition and actual vapor pressures. We found that at these expected temperatures, solid $\mathrm{H}_{2}$ would boil off immediately. The first discussion of $\mathrm{CN}$ as a possible radiometer in space was by Swings in 1939, at a time when nobody knew about black-body cosmological radiation. The first discussion on grain temperatures that I know of was by Eddington in 1923 . He made such calculations saying the total radiation field is roughly $10^{4} \mathrm{~K}$, and the dilution factor $10^{-14}$, so

$$
T_{\text {grain }}=10^{4} \times\left(10^{-14}\right)^{1 / 4}=3.2 \mathrm{~K} .
$$

Greenberg reminded you of the size effect, which can very roughly be taken into account by simply replacing the exponent by $\frac{1}{5}$, because there is a $1 / \lambda$ dependence. This makes

$$
T_{\text {grain }}=10^{4} \times\left(10^{-14}\right)^{1 / 5}=10 \mathrm{~K} .
$$

(Oort, J. H. and van de Hulst, H. C.: 1946, Bull. Astron. Inst. Neth. 10, 187.) 